\title{
COMPUTER-AIDED TONGUE IMAGE DIAGNOSIS AND ANALYSIS
}

\author{
Ratchadaporn Kanawong \\ Dr. Ye Duan, Dissertation Supervisor
}

\begin{abstract}
This work focuses on computer-aided tongue image analysis, specifically, as it relates to Traditional Chinese Medicine (TCM). Computerized tongue diagnosis aid medical practitioners capture quantitative features to improve reliability and consistence of diagnosis. A total computer-aided tongue analysis framework consists of tongue detection, tongue segmentation, tongue feature extraction, tongue classification and analysis, which are all included in our work.

We propose a new hybrid image segmentation algorithm that integrates the regionbased method with the boundary-based method. We apply this segmentation algorithm in designing an automatic tongue detection and segmentation framework. We also develop a novel color space based feature set for tongue feature extraction to implement an automated ZHENG (TCM syndrome) classification system using machine learning techniques. To further enhance the performance of our classification system, we propose preprocessing the tongue images using the Modified Specular-free technique prior to feature extraction, and explore the extraction of geometry features from the Petechia. Lastly, we propose a new feature set for automated tongue shape classification.
\end{abstract}

